\title{
WATER RESOURCES OF THE RIVER ROUGE BASIN, SOUTHEASTERN MICHIGAN
}

\begin{abstract}
By
R. L. Knutilla

1970

\section{PHYSIOGRAPHIC DATA FOR SELECTED LOCATIONS IN THE RIVER ROUGE BASIN}

The accompanying tables summarize some physiographic data for selected locations on streams in the River Rouge basin having about 2 or more square miles of drainage area. Selected locations on the major streams include locations above mouths of tributaries, and points of interest such as highways, towns, gaging stations, or dams, and below the confluence of the main stream and tributary so as to include both drainage areas. Similar criteria were used for tributary streams except where the tributary was too small to merit further dividing. The divided areas are shown on the basin map, sheet 1 . To help locate the tabulated areas on the map, each location is assigned a number and numbers for selected sites are shown on the map. Also shown are names of main roads and river miles, at 5-mile intervals, for the River Rouge and its three main tributaries.

Basin divides were outlined on the latest $71 / 2$-minute topographic maps, scale 1:24,000, and drainage areas determined in accordance with the standards set forth by the Committee on Hydrology, Water Resources Council (formerly the Subcommittee on Hydrology, Inter-agency Committee on Water Resources). Similarly, river miles were determined in accordance with the Committee's standards. Mile zero is considered to be the mouth of the stream. The source of a stream is considered to be the upper end of the stream, whether or not it is perennial. A defined

channel which may be inferred from topographic maps to carry water occasionally is, therefore, included in the stream length. A stream that has its beginning at the confluence of two tributaries is considered to be a continuation of the longer tributary and to have as its source the source of that tributary. Its average slope is determined by treating the stream and tributary as one. River Rouge, for example, is considered to be the continuation of Sprague Ditch. In the table Sprague Ditch has been parenthetically noted as being the headwaters of River Rouge. Other tributary streams, which fall into this classification, are likewise noted.

Altitudes shown for site and source are those obtained from topographic maps and represent the altitude of the water surface at approximately normal river stage, the datum being mean sea level. Average slopes were computed from the mileage between and differences in altitude of site and source and represent the fall of the stream in feet per mile.

Streams are listed in a downstream order along the main stem, with tributary streams entered above the next main-stem location. A similar order is followed for listing streams of first rank, second rank, or other rank. To indicate the rank, and the stream to which it is tributary, the stream name is indented, each indentation representing one rank. The stream rank is further identified by numbers in the column heading with stream name and location. Number 1 applies to River Rouge. Principal tributaries are under number 2, their tributaries under number 3 , and so on.
\end{abstract}

PHYSIOGRAPHIC DATA FOR SELECTED LOCATIONS IN THE RIVER ROUGE BASIN

\begin{tabular}{|c|c|c|c|c|c|c|c|}
\hline \multirow{2}{*}{$\begin{array}{l}\text { Num- } \\
\text { ber } \\
\text { Key }\end{array}$} & Stream and location & \multirow{2}{*}{$\begin{array}{l}\text { Drainage } \\
\text { area } \\
\text { (sq mi) }\end{array}$} & \multicolumn{2}{|c|}{ Miles } & \multicolumn{2}{|c|}{$\begin{array}{c}\text { Altitude } \\
\text { (ft above msl) }\end{array}$} & \multirow{2}{*}{$\begin{array}{c}\text { Average } \\
\text { slope } \\
\text { (ft per } \\
\text { mile) }\end{array}$} \\
\hline & Ranking order & & $\begin{array}{l}\text { above } \\
\text { mouth }\end{array}$ & $\begin{array}{l}\text { below } \\
\text { source }\end{array}$ & $\begin{array}{l}\text { at } \\
\text { site }\end{array}$ & $\begin{array}{c}\text { at } \\
\text { source }\end{array}$ & \\
\hline 1. & $\begin{array}{l}\text { Sprague Ditch (headwaters of River Rouge) } \\
\text { above Sprague Branch, } \\
\mathrm{SW}^{1 / 4} \text { sec. 5.T. } 2 \text { N., R. } 11 \mathrm{E} \text {. }\end{array}$ & 2.83 & 44.4 & 1.2 & 792 & 798 & 5.0 \\
\hline 2. & $\begin{array}{l}\text { Sprague Branch at mouth, } \\
\text { SW1/4 sec. 5, T. } 2 \text { N., R. } 11 \text { E. }\end{array}$ & 3.98 & 0 & 1.2 & 794 & 820 & 23.3 \\
\hline 3. & $\begin{array}{l}\text { Sprague Ditch at Sprague Branch, } \\
\text { SW1/4 sec. 5, T.2 N., R. } 11 \text { E. }\end{array}$ & 6.81 & 44.4 & 1.2 & 794 & 798 & 5.0 \\
\hline & NE $1 / 4$ sec. 24, T. 2 N., R. 10 E. & 12.0 & 41.1 & 4.5 & 752 & 798 & 10.2 \\
\hline
\end{tabular}


PHYSIOGRAPHIC DATA FOR SELECTED LOCATIONS IN THE RIVER ROUGE BASIN

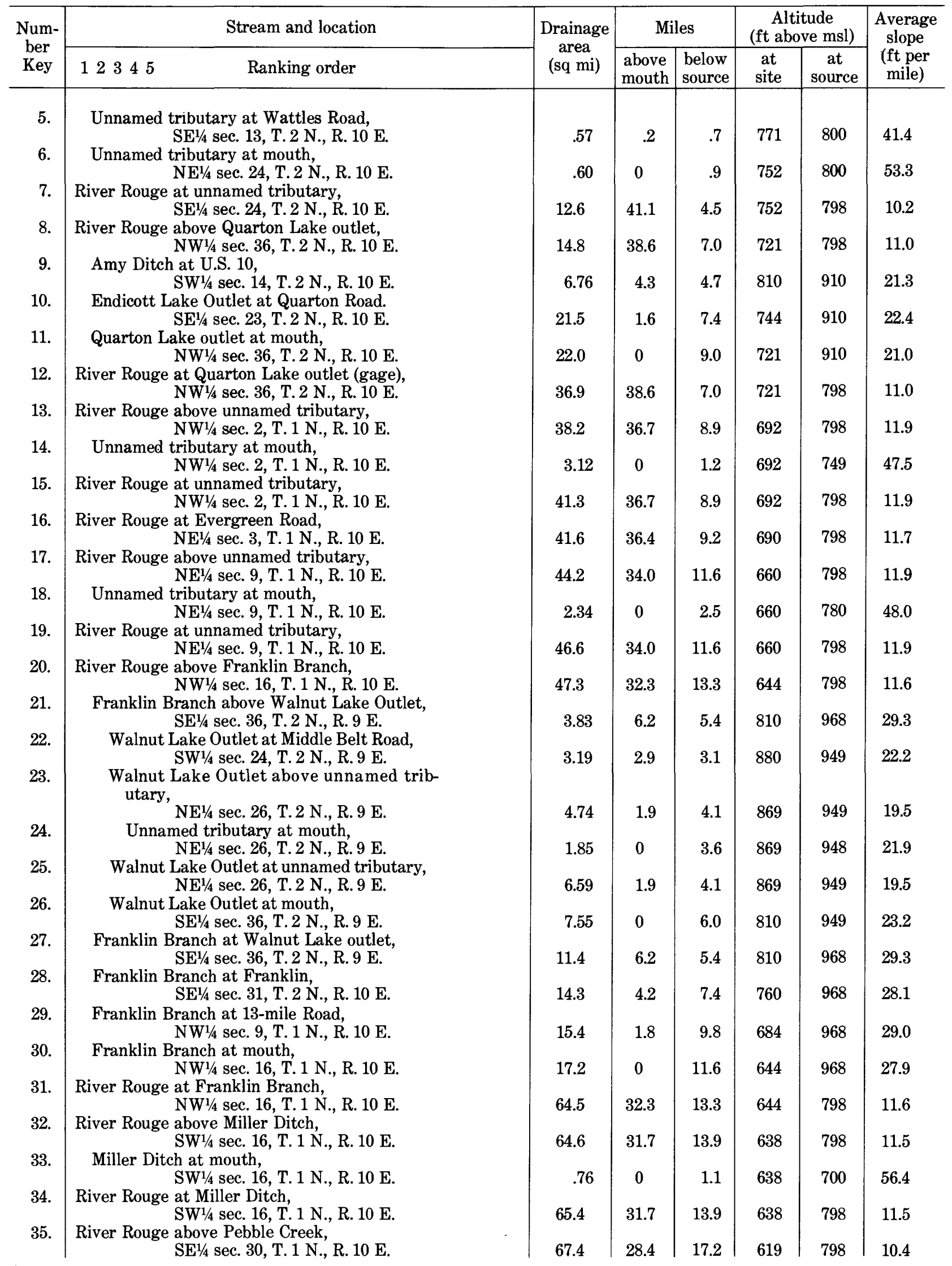


PHYSIOGRAPHIC DATA FOR SELECTED LOCATIONS IN THE RIVER ROUGE BASIN

\begin{tabular}{|c|c|c|c|c|c|c|c|}
\hline \multirow{2}{*}{$\begin{array}{l}\text { Num- } \\
\text { ber } \\
\text { Key }\end{array}$} & Stream and location & \multirow{2}{*}{$\begin{array}{l}\text { Drainage } \\
\text { area } \\
\text { (sq mi) }\end{array}$} & \multicolumn{2}{|c|}{ Miles } & \multicolumn{2}{|c|}{$\begin{array}{c}\text { Altitude } \\
\text { (ft above msl) }\end{array}$} & \multirow{2}{*}{$\begin{array}{c}\text { Average } \\
\text { slope } \\
\text { (ft per } \\
\text { mile) }\end{array}$} \\
\hline & Ranking order & & $\begin{array}{l}\text { above } \\
\text { mouth }\end{array}$ & \begin{tabular}{|l|} 
below \\
source
\end{tabular} & $\begin{array}{l}\text { at } \\
\text { site }\end{array}$ & $\begin{array}{c}\text { at } \\
\text { source }\end{array}$ & \\
\hline 36. & Pebble Creek at Middle Belt Road, & & & & & & \\
\hline 37. & $\begin{array}{l}\mathrm{NE}^{1 / 4} \text { sec. } 11, \mathrm{~T} .1 \text { N., R. } 9 \text { E. } \\
\text { Pebble Creek above Lake Genesareth outlet. }\end{array}$ & 4.07 & 5.2 & 5.1 & 806 & 993 & 36.7 \\
\hline & & 6.39 & 1.2 & 9.1 & 637 & 993 & 39.1 \\
\hline 38. & $\begin{array}{l}\text { Lake Genesareth outlet at mouth, } \\
\text { SE1/4 sec. } 19, \text { T. } 1 \text { N., R. } 10 \text { E. }\end{array}$ & 3.87 & 0 & 4.3 & 637 & 794 & 36.5 \\
\hline 39. & $\begin{array}{l}\text { Pebble Creek at Lake Genesareth outlet, } \\
\mathrm{SE}^{1 / 4} \text { sec. } 19 \text {, T. } 1 \text { N., R. } 10 \mathrm{E} \text {. }\end{array}$ & 10.3 & 12 & 91 & 637 & & \\
\hline 40. & Pebble Creek at 10 -mile road, & 10.0 & & & 637 & 993 & 39.1 \\
\hline 41. & $\begin{array}{l}\mathrm{SE}^{1 / 4} \text { sec. } 19, \text { T. } 1 \text { N., R. } 10 \mathrm{E} . \\
\text { Pebble Creek at mouth, }\end{array}$ & 10.3 & 1.1 & 9.2 & 635 & 993 & 38.9 \\
\hline 42. & $\begin{array}{l}\text { SE1/4 sec. } 30, \text { T. } 1 \text { N., R. } 10 \text { E. } \\
\text { River Rouge at Pebble Creek, }\end{array}$ & 11.0 & 0 & 10.3 & 619 & 993 & 36.3 \\
\hline 43. & $\begin{array}{l}\text { SE1/4 sec. } 30, \text { T. } 1 \text { N., R. } 10 \text { E. } \\
\text { River Rouge above unnamed tributary No. } 3 \text {, }\end{array}$ & 78.3 & 28.4 & 17.2 & 619 & 798 & 10.4 \\
\hline 44. & $\begin{array}{l}\text { NW1/4 sec. } 31, \mathrm{~T} .1 \mathrm{~N} ., \mathrm{R} .10 \mathrm{E} . \\
\text { Unnamed tributary No. } 3 \text { above unnamed trib- } \\
\text { utary No. } 2 \text {, }\end{array}$ & 78.6 & 27.6 & 18.0 & 616 & 798 & 10.1 \\
\hline 45. & $\begin{array}{l}\mathrm{SE}^{1 / 4} \text { sec. } 25, \mathrm{~T} .1 \text { N., R. } 9 \text { E. } \\
\text { Unnamed tributary No. } 2 \text { at mouth, }\end{array}$ & 3.80 & .2 & 4.2 & 623 & 845 & 52.9 \\
\hline 46. & $\begin{array}{l}\mathrm{SE}^{1 / 4} \text { sec. } 25, \mathrm{~T} .1 \text { N., R. } 9 \mathrm{E} \text {. } \\
\text { Unnamed tributary No. } 3 \text { at unnamed trib- } \\
\text { utary No. } 2 \text {, }\end{array}$ & 1.26 & 0 & 3.8 & 623 & 810 & 49.2 \\
\hline 47. & $\begin{array}{l}\mathrm{SE}^{1 / 4} \text { sec. } 25, \mathrm{~T} .1 \mathrm{~N} ., \mathrm{R} .9 \mathrm{E} . \\
\text { Unnamed tributary No. } 3 \text { above unnamed } \\
\text { tributary No. } 1 \text {, }\end{array}$ & 5.06 & .2 & 4.2 & 623 & 845 & 52.9 \\
\hline 48. & $\begin{array}{l}\text { NW1/4 sec. } 31, \text { T. } 1 \text { N., R. } 10 \text { E. } \\
\text { Unnamed tributary No. } 1 \text { at mouth, }\end{array}$ & 5.12 & .2 & 4.4 & 619 & 845 & 51.4 \\
\hline 49. & $\begin{array}{l}\text { NW1/4 sec. } 31, \mathrm{~T} .1 \mathrm{~N} ., \mathrm{R} .10 \mathrm{E} . \\
\text { Unnamed tributary No. } 3 \text { at unnamed trib- } \\
\text { utary No. } 1 \text {, }\end{array}$ & 3.46 & 0 & 3.8 & 619 & 802 & 48.2 \\
\hline 50. & $\begin{array}{l}\text { NW'1/4 sec. } 31, T .1 \text { N., R. } 10 \mathrm{E} . \\
\text { Unnamed tributary No. } 3 \text { at mouth, }\end{array}$ & 8.58 & .2 & 4.4 & 619 & 845 & 51.4 \\
\hline 51. & $\begin{array}{l}\text { NW } 1 / 4 \text { sec. } 31, T .1 \text { N., R. } 10 \text { E. } \\
\text { River Rouge at unnamed tributary No. } 3\end{array}$ & 8.64 & 0 & 4.6 & 616 & 845 & 49.8 \\
\hline 52. & $\begin{array}{l}\text { NW1/4 sec. 31, T. } 1 \text { N., R. } 10 \text { E. } \\
\text { River Rouge at Beech Road (gage), }\end{array}$ & 87.2 & 27.6 & 18.0 & 616 & 798 & 10.1 \\
\hline 53. & $\begin{array}{l}\text { SW1/4 sec. 32, T.1 N., R. } 10 \text { E. } \\
\text { River Rouge at Oakland County line, }\end{array}$ & 87.9 & 26.5 & 19.1 & 611 & 798 & 9.8 \\
\hline 54. & $\begin{array}{l}\mathrm{SE}^{1 / 4} \mathrm{sec} .32, \mathrm{~T} .1 \mathrm{~N} ., \mathrm{R} .10 \mathrm{E} . \\
\text { River Rouge above Evans Ditch, }\end{array}$ & 89.2 & 25.3 & 20.3 & 608 & 798 & 9.4 \\
\hline 55. & $\begin{array}{l}\text { NW1/4 sec. 4, T.1 S., R. } 10 \text { E. } \\
\text { Evans Ditch above McKinley Ditch, }\end{array}$ & 89.4 & 24.8 & 20.8 & 607 & 798 & 9.2 \\
\hline 56. & $\begin{array}{l}\text { NW'1/4 sec. } 27, \text { T. } 1 \text { N., R. } 10 \text { E. } \\
\text { McKinley Ditch at mouth, }\end{array}$ & 5.58 & 3.3 & 5.7 & 635 & 740 & 18.4 \\
\hline & NW'1/4 sec. 27 , T. 1 N., R. 10 E. & 2.57 & 0 & 2.4 & 635 & 691 & 23.3 \\
\hline 57. & $\begin{array}{l}\text { Evans Ditch at McKinley Ditch, } \\
\text { NW1/4 sec. } 27 \text {, T. } 1 \text { N., R. } 10 \text { E. }\end{array}$ & 8.15 & 3.5 & 5.7 & 635 & 740 & 18.4 \\
\hline 58. & $\begin{array}{l}\text { Evans Ditch at 9-mile Road (gage), } \\
\text { SE } 1 / 4 \text { sec. } 28, T .1 \text { N., R. } 10 \text { E. }\end{array}$ & 9.49 & 1.6 & 7.4 & 621 & 740 & 16.1 \\
\hline 59. & $\begin{array}{l}\text { Evans Ditch at Oakland County line, } \\
\text { SW11/4 sec. 33, T. } 1 \text { N., R. } 10 \text { E. }\end{array}$ & 10.0 & .4 & 8.6 & 610 & 740 & 15.1 \\
\hline 60. & $\begin{array}{l}\text { Evans Ditch at mouth, } \\
\qquad N^{1 / 4} \text { sec. } 4, \text { T. } 1 \text { S., R. } 10 \text { E. }\end{array}$ & 11.1 & 0 & 9.0 & 607 & 740 & 14.8 \\
\hline $\begin{array}{l}61 . \\
62 .\end{array}$ & $\begin{array}{l}\text { River Rouge at Evans Ditch, } \\
\text { NW11/4 sec. } 4 \text {, T. } 1 \text { S., R. } 10 \mathrm{E} . \\
\text { River Rouge above Griffin Drain }\end{array}$ & 100 & 24.8 & 20.8 & 607 & 798 & 9.2 \\
\hline 63. & $\begin{array}{l}\text { NW1/4 sec. 9, T. } 1 \text { S., R. } 10 \text { E. } \\
\text { Griffin Drain at Oakland County line, }\end{array}$ & 101 & 23.6 & 22.0 & 604 & 798 & 8.8 \\
\hline 64. & $\begin{array}{l}\mathrm{SE}^{1 / 4} \text { sec. 34, T. } 1 \text { N., R. } 10 \mathrm{E} . \\
\text { Griffin Drain above Morgan Ditch. }\end{array}$ & 1.60 & 2.4 & 3.0 & 649 & 689 & 13.3 \\
\hline & SW1/4 sec. 3, T. 1 S., R. 10 E. & 1.83 & 1.2 & 4.2 & 634 & 689 & 13.1 \\
\hline
\end{tabular}


PHYSIOGRAPHIC DATA FOR SELECTED LOCATIONS IN THE RIVER ROUGE BASIN

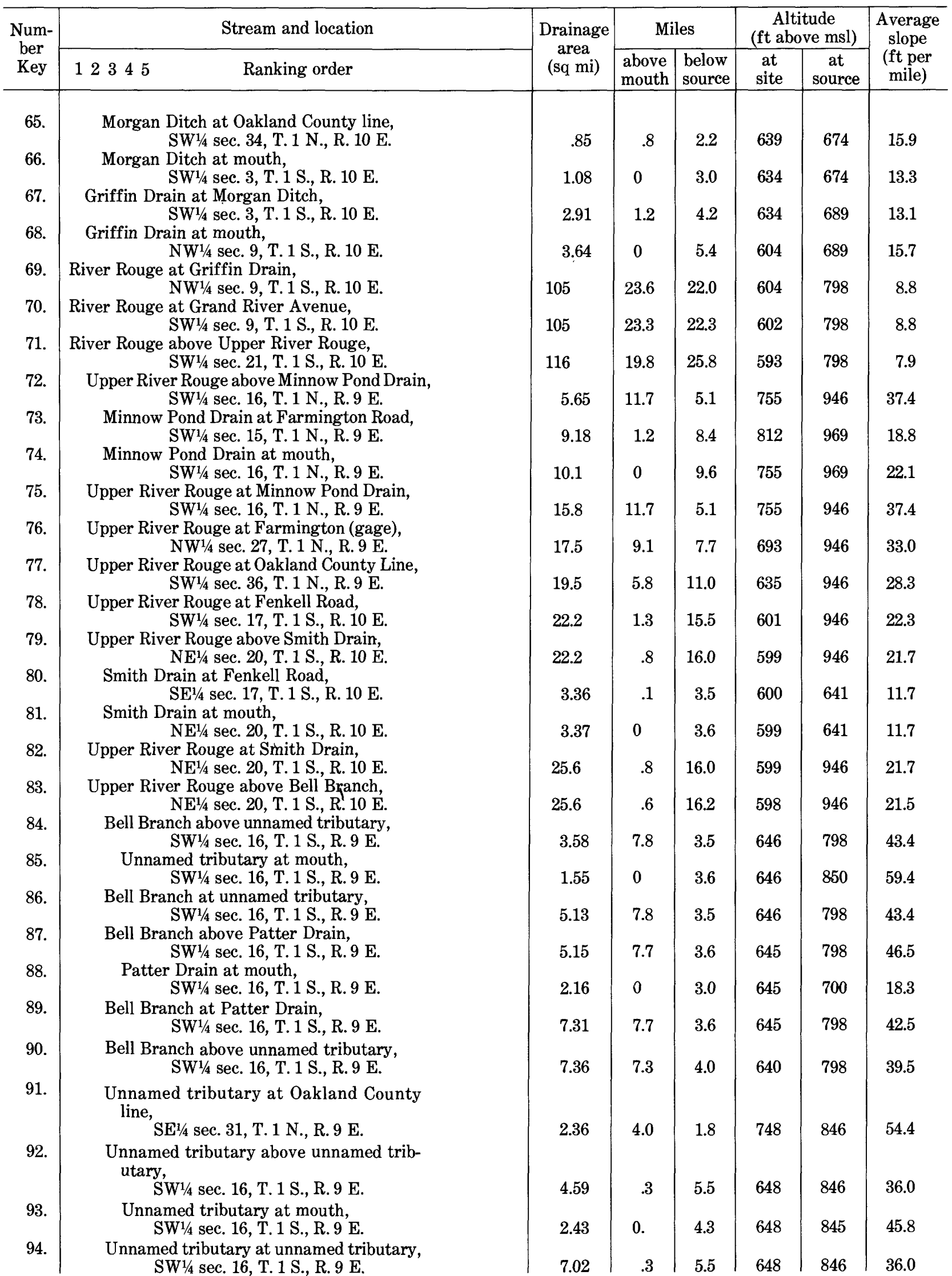


PHYSIOGRAPHIC DATA FOR SELECTED LOCATIONS IN THE RIVER ROUGE BASIN

\begin{tabular}{|c|c|c|c|c|c|c|c|}
\hline \multirow{2}{*}{$\begin{array}{l}\text { Num- } \\
\text { ber } \\
\text { Key }\end{array}$} & Stream and location & \multirow{2}{*}{$\begin{array}{l}\text { Drainage } \\
\text { area } \\
\text { (sq mi) }\end{array}$} & \multicolumn{2}{|c|}{ Miles } & \multicolumn{2}{|c|}{$\begin{array}{c}\text { Altitude } \\
\text { (ft above msl) }\end{array}$} & \multirow{2}{*}{$\begin{array}{c}\text { Average } \\
\text { slope } \\
\text { (ft per } \\
\text { mile) }\end{array}$} \\
\hline & Ranking order & & $\begin{array}{l}\text { above } \\
\text { mouth }\end{array}$ & \begin{tabular}{|l|} 
below \\
source
\end{tabular} & $\begin{array}{l}\text { at } \\
\text { site }\end{array}$ & $\begin{array}{c}\text { at } \\
\text { source }\end{array}$ & \\
\hline 95. & $\begin{array}{l}\text { Unnamed tributary at mouth, } \\
\text { SW1/4 sec. } 16 \text {, T. } 1 \text { S., R. } 9 \mathrm{E} .\end{array}$ & 7.06 & 0 & 5.8 & 640 & 846 & 35.5 \\
\hline $\begin{array}{l}96 . \\
97 .\end{array}$ & $\begin{array}{l}\text { Bell Branch at unnamed tributary, } \\
\mathrm{SW}^{1 / 4} \text { sec. } 16, \mathrm{~T} .1 \mathrm{~S} ., \mathrm{R} .9 \mathrm{E} . \\
\text { Bell Branch above Gates Drain, }\end{array}$ & 14.4 & 7.3 & 4.0 & 640 & 798 & 39.5 \\
\hline 98. & $\begin{array}{l}\mathrm{SW}^{1 / 4} \text { sec. } 16 \text {, T. } 1 \mathrm{~S} ., \mathrm{R} .9 \mathrm{E} . \\
\text { Gates Drain at mouth, }\end{array}$ & 14.4 & 7.1 & 4.2 & 639 & 798 & 37.9 \\
\hline 99. & $\begin{array}{l}\text { SW1/4 sec. } 16 \text {, T. } 1 \text { S., R. } 9 \text { E. } \\
\text { Bell Branch at Gates Drain, }\end{array}$ & .42 & 0 & 1.4 & 639 & 661 & 15.7 \\
\hline 100. & $\begin{array}{l}\text { SW11/4 sec. } 16 \text {, T. } 1 \mathrm{~S} \text {., R. } 9 \mathrm{E} \text {. } \\
\text { Bell Branch above unnamed tributary }\end{array}$ & 14.9 & 7.1 & $4: 2$ & 639 & 798 & 37.9 \\
\hline 101. & $\begin{array}{l}\mathrm{NE}^{1 / 4} \text { sec. } 16, \mathrm{~T} .1 \mathrm{~S} ., \mathrm{R} .9 \mathrm{E} . \\
\text { Unnamed tributary at mouth, }\end{array}$ & 15.1 & 6.7 & 4.6 & 636 & 798 & 35.2 \\
\hline 102 & $\begin{array}{l}\text { NE } 1 / 4 \text { sec. } 16 \text {, T.1 S., R. } 9 \text { E. } \\
\text { Bell }\end{array}$ & 2.72 & 0 & 5.7 & 636 & 847 & 37.0 \\
\hline 103. & $\begin{array}{l}\text { NE } 1 / 4 \text { sec. 16, T. } 1 \text { S., R. } 9 \text { E. } \\
\text { Bell Branch above Barlow Drain, }\end{array}$ & 17.9 & 6.7 & 4.6 & 636 & 798 & 35.2 \\
\hline 104. & $\begin{array}{l}\text { SE1/4 sec. 16, T. } 1 \text { S., R. } 9 \text { E. } \\
\text { Barlow Drain at mouth, }\end{array}$ & 17.9 & 6.4 & 4.9 & 634 & 798 & 33.5 \\
\hline 105. & $\begin{array}{l}\mathrm{SE}^{1 / 4} \text { sec. 16, T. } 1 \mathrm{~S} ., \text { R. } 9 \mathrm{E} \text {. } \\
\text { Bell Branch at Barlow Drain, }\end{array}$ & .54 & 0 & 1.6 & 634 & 649 & 15.6 \\
\hline 106. & $\begin{array}{l}\mathrm{SE}^{1 / 4} \text { sec. } 16, \mathrm{~T} .1 \mathrm{~S} ., \mathrm{R} .9 \mathrm{E} . \\
\text { Bell Branch at Middle Belt Drain, }\end{array}$ & 18.5 & 6.4 & 4.9 & 634 & 798 & 33.5 \\
\hline 107. & $\begin{array}{l}\mathrm{NE}^{1 / 4} \text { sec. } 14, \mathrm{~T} .1 \mathrm{~S} \text {., R. } 9 \mathrm{E} . \\
\text { Bell Branch above Wolfrom Drain, }\end{array}$ & 20.2 & 3.8 & 7.5 & 617 & 798 & 24.1 \\
\hline 108. & $\begin{array}{l}\mathrm{NW}^{1 / 4} \text { sec. } 13, \mathrm{~T} .1 \mathrm{~S} ., \text { R. } 9 \mathrm{E} . \\
\text { Wolfrom Drain at mouth, }\end{array}$ & 20.3 & 3.5 & 7.8 & 615 & 798 & 23.5 \\
\hline 109. & $\begin{array}{l}\text { NW } 1 / 4 \text { sec. } 13 \text {, T.1 S., R. } 9 \text { E. } \\
\text { Bell Branch at Wolfrom Drain, }\end{array}$ & .54 & 0 & 1.2 & 615 & 640 & 20.8 \\
\hline 110. & $\begin{array}{l}\text { NW1/4 sec. 13, T. } 1 \text { S., R. } 9 \text { E. } \\
\text { Bell Branch above Tarabusi Creek. }\end{array}$ & 20.8 & 3.5 & 7.8 & 615 & 798 & 23.5 \\
\hline 111. & $\begin{array}{l}\text { NW1/4 sec. 13, T.1 S., R. } 9 \text { E. } \\
\text { Tarabusi Creek at Oakland County line, }\end{array}$ & 20.9 & 3.4 & 7.9 & 614 & 798 & 23.3 \\
\hline 112. & $\begin{array}{l}\mathrm{SE}^{1 / 4} \text { sec. 33, T. } 1 \text { N., R. } 9 \mathrm{E} . \\
\text { Tarabusi Creek at Middle Belt Road, }\end{array}$ & 4.52 & 4.2 & 5.4 & 693 & 851 & 29.3 \\
\hline 113. & $\begin{array}{l}\mathrm{SE}^{1 / 4} \text { sec. 11, T. } 1 \text { S., R. } 9 \text { E. } \\
\text { Tarabusi Creek at mouth. }\end{array}$ & 6.68 & .8 & 8.8 & 622 & 851 & 26.0 \\
\hline 114. & $\begin{array}{l}\text { NW1/4 sec. } 13, \text { T. } 1 \mathrm{~S} ., \text { R. } 9 \text { E. } \\
\text { Bell Branch at Tarabusi Creek. }\end{array}$ & 6.88 & 0 & 9.6 & 614 & 851 & 24.7 \\
\hline 115. & $\begin{array}{c}\text { NW1/4 sec. 13, T. 1 S., R. } 9 \text { E. } \\
\text { Bell Branch above unnamed tributary, }\end{array}$ & 27.8 & 3.4 & 7.9 & 614 & 798 & 23.3 \\
\hline 116. & $\begin{array}{l}\mathrm{NE}^{1 / 4} \text { sec. } 13, \mathrm{~T} .1 \mathrm{~S} ., \mathrm{R} .9 \mathrm{E} . \\
\text { Unnamed tributary at mouth, }\end{array}$ & 28.1 & 2.8 & 8.5 & 610 & 798 & 20.9 \\
\hline & $\begin{array}{l}\mathrm{NE}^{1 / 4} \text { sec. } 13, \mathrm{~T} .1 \mathrm{~S} ., \mathrm{R} .9 \mathrm{E} \text {. } \\
\text { Bell Branch at unnamed tributary, }\end{array}$ & 4.52 & 0 & 6.0 & 610 & 793 & 27.2 \\
\hline 110 & NE $1 / 4$ sec. 13, T. 1 S., R. 9 E. & 32.6 & 2.8 & 8.5 & 610 & 798 & 20.9 \\
\hline 118. & $\begin{array}{l}\text { Bell Branch above Bell Drain, } \\
\text { NW1/4 sec. } 19, \text { T. } 1 \text { S., R. } 10 \text { E. }\end{array}$ & 33.4 & 1.7 & 9.6 & 604 & 798 & 20.2 \\
\hline 119. & $\begin{array}{l}\text { Bell Drain at mouth, } \\
\text { NW1/4 sec. } 19, \text { T. } 1 \text { S., R. } 10 \text { E. }\end{array}$ & 2.49 & 0 & 3.6 & 604 & 655 & 14.2 \\
\hline 120. & $\begin{array}{l}\text { Bell Branch at Bell Drain, } \\
\text { NW1/4 sec. } 19 \text {, T. } 1 \text { S., R. } 10 \text { E. }\end{array}$ & 35.8 & 1.7 & 9.6 & 604 & 798 & 20.2 \\
\hline 121. & 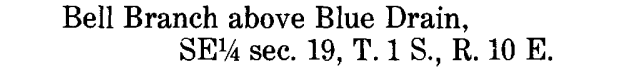 & 36.0 & 1.3 & 10.0 & 603 & 798 & 19.5 \\
\hline 122. & $\begin{array}{l}\text { Blue Drain at mouth, } \\
\text { SE } 1 / 4 \text { sec. } 19 \text {, T. } 1 \text { S., R. } 10 \text { E. }\end{array}$ & 1.70 & 0 & 4.2 & 603 & 649 & 11.0 \\
\hline 123. & $\begin{array}{l}\text { Bell Branch at Blue Drain, } \\
\text { SE } 1 / 4 \text { sec. } 19 \text {, T. } 1 \text { S., R. } 10 \text { E. }\end{array}$ & 37.7 & 1.3 & 10.0 & 603 & 798 & 19.5 \\
\hline 124. & $\begin{array}{l}\text { Bell Branch above Shaw Drain, } \\
\text { SE }^{1 / 4} \text { sec. } 19, \text { T. } 1 \text { S., R. } 10 \text { E. }\end{array}$ & 37.7 & 1.1 & 10.2 & 602 & 798 & 19.2 \\
\hline 125. & 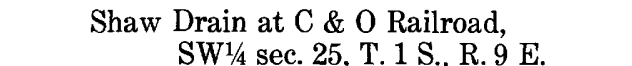 & .85 & 2.2 & 2.3 & 626 & 642 & 7.0 \\
\hline
\end{tabular}


PHYSIOGRAPHIC DATA FOR SELECTED LOCATIONS IN THE RIVER ROUGE BASIN

\begin{tabular}{|c|c|c|c|c|c|c|c|}
\hline \multirow{2}{*}{$\begin{array}{l}\text { Num- } \\
\text { ber } \\
\text { Key }\end{array}$} & Stream and location & \multirow{2}{*}{$\begin{array}{l}\text { Drainage } \\
\text { area } \\
\text { (sq mi) }\end{array}$} & \multicolumn{2}{|c|}{ Miles } & \multicolumn{2}{|c|}{$\begin{array}{c}\text { Altitude } \\
\text { (ft above msl) }\end{array}$} & \multirow{2}{*}{$\begin{array}{l}\text { Average } \\
\text { slope } \\
\text { (ft per } \\
\text { mile) }\end{array}$} \\
\hline & Ranking order & & $\begin{array}{l}\text { above } \\
\text { mouth }\end{array}$ & $\begin{array}{l}\text { below } \\
\text { source }\end{array}$ & $\begin{array}{c}\text { at } \\
\text { site }\end{array}$ & $\begin{array}{c}\text { at } \\
\text { source } \\
\end{array}$ & \\
\hline 126. & $\begin{array}{l}\text { Shaw Drain above Hawkins Drain, } \\
\text { SW1/4 sec. 25. T. } 1 \mathrm{~S} . \mathrm{R} .9 \mathrm{E}\end{array}$ & 1.10 & 2.1 & 2.4 & 625 & 642 & 7.1 \\
\hline 127. & $\begin{array}{l}\text { Hawkins Drain at mouth, } \\
\text { SW11/4 sec. } 25, \text { T. } 1 \text { S., R. } 9 \text { E. }\end{array}$ & .92 & 0 & 2.0 & 625 & $640^{\circ}$ & 7.5 \\
\hline 128. & $\begin{array}{l}\text { Shaw Drain at Hawkins Drain, } \\
\text { SW1/4 sec. } 25, \text { T. } 1 \text { S., R. } 9 \text { E }\end{array}$ & 2.02 & 2.1 & 2.4 & 625 & 642 & 7.1 \\
\hline 129. & 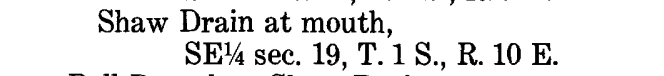 & 3.08 & 0 & 4.5 & 602 & 642 & 8.9 \\
\hline 130. & $\begin{array}{l}\text { Bell Branch at Shaw Drain, } \\
\text { SE}^{1 / 4} \text { sec. } 19, \text { T. } 1 \text { S., R. } 10 \text { E. }\end{array}$ & 40.8 & 1.1 & 10.2 & 602 & 798 & 19.2 \\
\hline 131. & $\begin{array}{l}\text { Bell Branch at Beech-Daly Road, } \\
\text { SEE }^{1 / 4} \text { sec. } 19, \text { T. } 1 \text { S., R. } 10 \text { E. }\end{array}$ & 40.8 & 1.0 & 10.3 & 602 & 798 & 19.0 \\
\hline 132. & $\begin{array}{l}\text { Bell Branch at mouth, } \\
\text { NE1/4 sec. } 20, \text { T. } 1 \text { S., R. } 10 \text { E. }\end{array}$ & 41.5 & 0 & 11.3 & 598 & 798 & 17.7 \\
\hline 133. & $\begin{array}{l}\text { Upper River Rouge at Bell Branch, } \\
\text { NE } 1 / 4 \text { sec. } 20 \text {, T. } 1 \text { S., R. } 10 \text { E. }\end{array}$ & 67.2 & .6 & 16.2 & 598 & 946 & 21.5 \\
\hline 134. & $\begin{array}{l}\text { Upper River Rouge at mouth, } \\
\text { SW1/4 sec. } 21 \text {, T. } 1 \text { S., R. } 10 \text { E. }\end{array}$ & 67.5 & 0 & 16.8 & 593 & 946 & 21.0 \\
\hline 135. & $\begin{array}{l}\text { River Rouge at Upper River Rouge, } \\
\text { SW1/4 sec. } 21 \text {, T. } 1 \text { S., R. } 10 \text { E. }\end{array}$ & 184 & 19.8 & 25.8 & 593 & 798 & 7.9 \\
\hline 136. & $\begin{array}{l}\text { River Rouge at Plymouth Road (gage), } \\
\text { SE1/4 sec. } 27, \text { T. } 1 \text { S., R. } 10 \text { E. }\end{array}$ & 187 & 17.1 & 28.5 & 588 & 798 & 7.4 \\
\hline 137. & $\begin{array}{l}\text { River Rouge near Joy Road (former gage), } \\
\text { SW1/4 sec. } 34 \text {, T. } 1 \text { S., R. } 10 \text { E. }\end{array}$ & 188 & 15.7 & 29.9 & 585 & 798 & 7.1 \\
\hline 138. & $\begin{array}{l}\text { River Rouge above Ashcroft Drain, } \\
\text { SE } 1 / 4 \text { sec. 33, T. } 1 \text { S., R. } 10 \text { E. }\end{array}$ & 188 & 15.4 & 30.2 & 585 & 798 & 7.1 \\
\hline 139. & $\begin{array}{l}\text { Ashcroft Drain at Inkster Road, } \\
\text { NE } 1 / 4 \text { sec. 36, T. 1 S., R. } 9 \text { E. }\end{array}$ & 1.04 & 3.2 & 2.0 & 623 & 635 & 6.0 \\
\hline 140. & 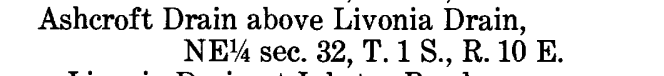 & 1.97 & 1.6 & 3.6 & 605 & 635 & 8.3 \\
\hline 141. & $\begin{array}{l}\text { Livonia Drain at Inkster Road, } \\
\mathrm{SE}^{1 / 4} \text { sec. } 25, \mathrm{~T} .1 \text { S., R. } 9 \text { E. }\end{array}$ & .74 & 1.7 & 2.1 & 623 & 635 & 5.7 \\
\hline 142. & $\begin{array}{l}\text { Livonia Drain at mouth, } \\
\text { NE } 1 / 4 \text { sec. } 32 \text {, T. } 1 \text { S., R. } 10 \text { E. }\end{array}$ & 1.17 & 0 & 3.8 & 605 & 635 & 7.9 \\
\hline 143. & $\begin{array}{l}\text { Asheroft Drain at Livonia Drain, } \\
\text { NE } 1 / 4 \text { sec. } 32 \text {, T. } 1 \text { S., R. } 10 \text { E. }\end{array}$ & 3.14 & 1.6 & 3.6 & 605 & 635 & 8.3 \\
\hline 144. & $\begin{array}{l}\text { Ashcroft Drain above Prindle Drain, } \\
\text { NEE}^{1 / 4} \text { sec. } 32 \text {, T. } 1 \text { S., R. } 10 \text { E. }\end{array}$ & 3.20 & 1.3 & 3.9 & 603 & 635 & 8.2 \\
\hline 145. & $\begin{array}{l}\text { Prindle Drain at mouth, } \\
\text { NE1/4 sec. } 32 \text {, T. } 1 \text { S., R. } 10 \text { E. }\end{array}$ & .93 & 0 & 1.1 & 603 & 621 & 16.4 \\
\hline 146. & $\begin{array}{l}\text { Ashcroft Drain at Prindle Drain, } \\
\text { NE } 1 / 4 \text { sec. } 32 \text {, T. } 1 \text { S., R. } 10 \text { E. }\end{array}$ & 4.13 & 1.3 & 3.9 & 603 & 635 & 8.2 \\
\hline 147. & $\begin{array}{l}\text { Asheroft Drain at mouth, } \\
\text { SE1/4 sec. } 33, \text { T. } 1 \text { S., R. } 10 \text { E. }\end{array}$ & 5.31 & 0 & 5.2 & 585 & 635 & 9.6 \\
\hline 148. & $\begin{array}{l}\text { River Rouge at Ashcroft Drain, } \\
\mathrm{SE}^{1 / 4} \mathrm{sec} \text {. } 33 \text {, T. } 1 \mathrm{~S} \text {., R. } 10 \mathrm{E} \text {. }\end{array}$ & 194 & 15.4 & 30.2 & 585 & 798 & 7.1 \\
\hline 149. & $\begin{array}{l}\text { River Rouge above Middle River Rouge, } \\
\text { SW1/4 sec. } 10 \text {, T. } 2 \text { S., R. } 10 \text { E. }\end{array}$ & 197 & 12.4 & 33.2 & 584 & 798 & 6.4 \\
\hline 150. & $\begin{array}{l}\text { Walled Lake Branch (headwaters of Middle } \\
\text { River Rouge) at Grand Rapids Avenue, } \\
\text { SE1/4 sec. 15, T. } 1 \text { N., R. } 8 \text { E. }\end{array}$ & 8.75 & 28.1 & 3.1 & 898 & 933 & 11.3 \\
\hline 151. & $\begin{array}{l}\text { Walled Lake Branch at Novi Road, } \\
\text { NE1/4 sec. } 22 \text {, T. } 1 \text { N., R. } 8 \text { E. } \\
\text { Walled Lake Branch above Ingersoll Creek, }\end{array}$ & 9.75 & 27.6 & 3.6 & 885 & 933 & 13.3 \\
\hline 153. & $\begin{array}{l}\text { SE1/4 sec. 26, T. } 1 \text { N., R. } 8 \text { E. } \\
\text { Ingersoll Creek above Bishop Creek, } \\
\text { NW1/4 sec. } 25 \text {, T. } 1 \text { N., R. } 8 \text { E. } \\
\text { Bishop Creek at mouth, }\end{array}$ & 3.64 & 25.4 & 5.8 & 831 & 933 & 17.8 \\
\hline 155. & $\begin{array}{l}\text { NW1/4 sec. } 25 \text {, T. } 1 \text { N., R. } 8 \text { E. } \\
\text { Ingersoll Creek at Bishop Creek, }\end{array}$ & 2.12 & 0 & 2.4 & 837 & 894 & 23.8 \\
\hline 156. & $\begin{array}{l}\mathrm{NW}^{1 / 4} \text { sec. } 25, \mathrm{~T} .1 \mathrm{~N} \text {., R. } 8 \mathrm{E} . \\
\text { Ingersoll Creek at mouth, } \\
\mathrm{SE}^{1 / 4} \text { sec. } 26, \mathrm{~T} .1 \text { N., R. } 8 \text { E. }\end{array}$ & 5.76 & .6 & 2.3 & 837 & 878 & 17.8 \\
\hline
\end{tabular}


PHYSIOGRAPHIC DATA FOR SELECTED LOCATIONS IN THE RIVER ROUGE BASIN

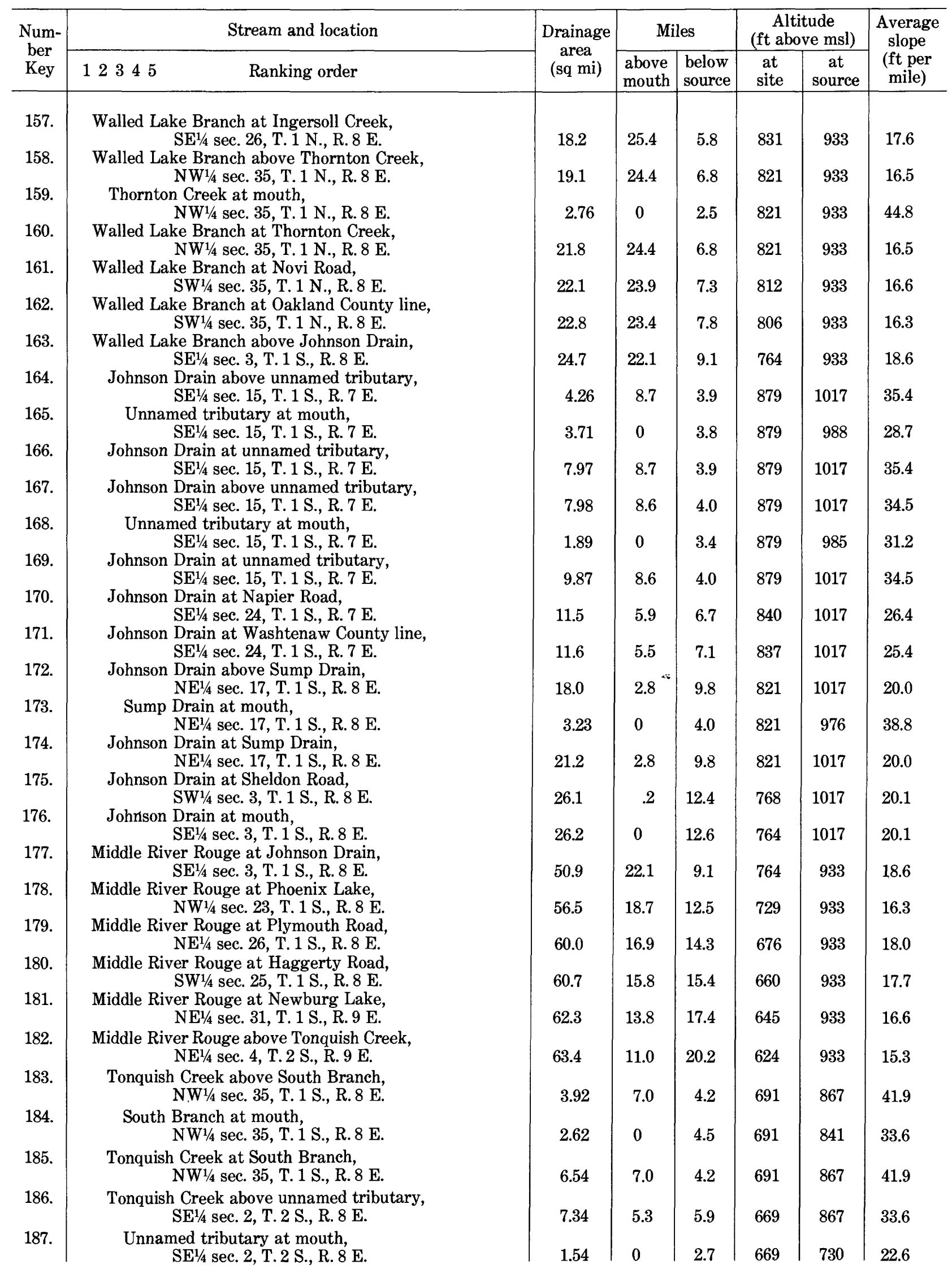


PHYSIOGRAPHIC DATA FOR SELECTED LOCATIONS IN THE RIVER ROUGE BASIN

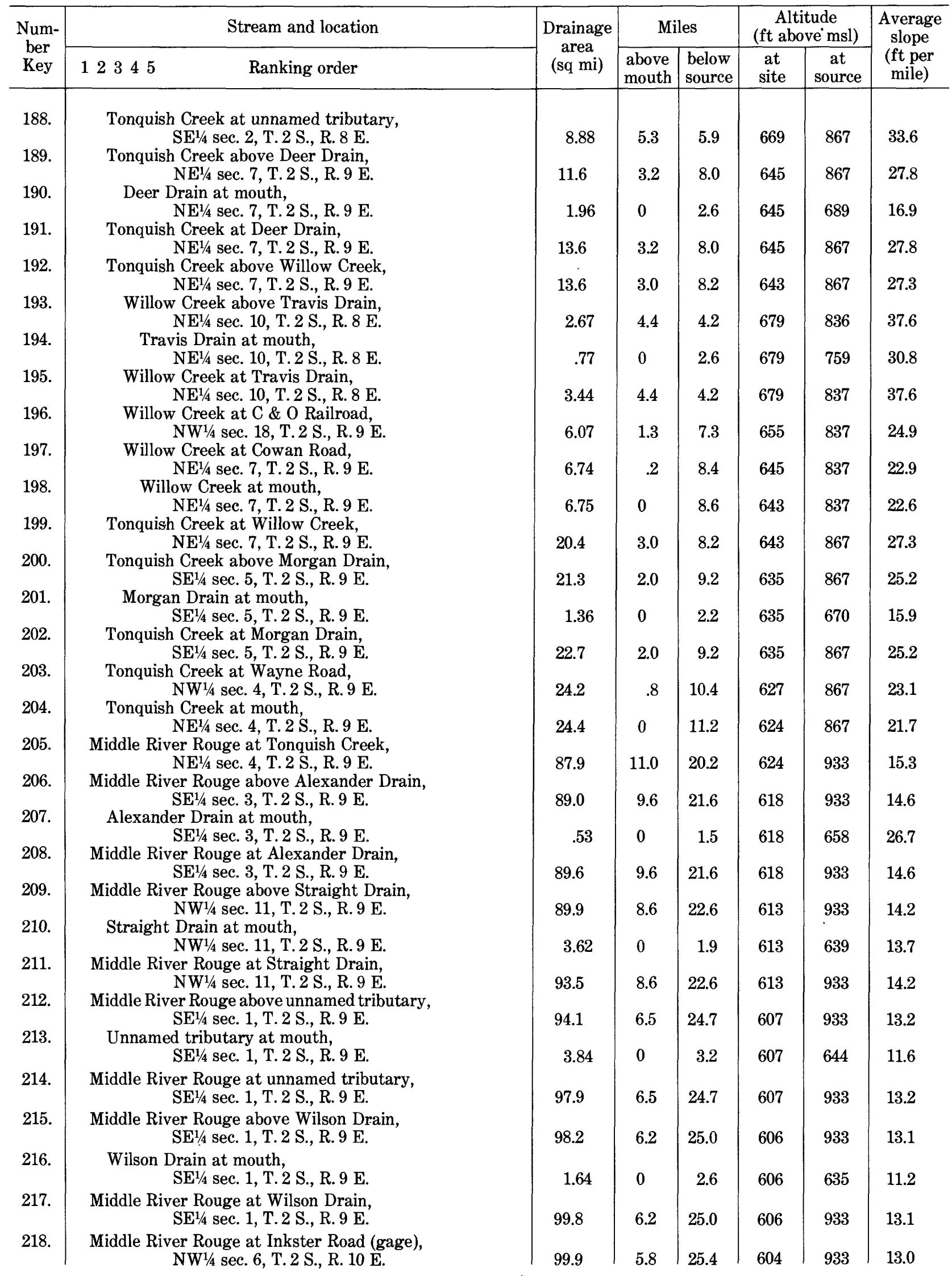


PHYSIOGRAPHIC DATA FOR SELECTED LOCATIONS IN THE RIVER ROUGE BASIN

\begin{tabular}{|c|c|c|c|c|c|c|c|}
\hline \multirow{2}{*}{$\begin{array}{l}\text { Num- } \\
\text { ber } \\
\text { Key }\end{array}$} & Stream and location & \multirow{2}{*}{$\begin{array}{l}\text { Drainage } \\
\text { area } \\
\text { (sq mi) }\end{array}$} & \multicolumn{2}{|c|}{ Miles } & \multicolumn{2}{|c|}{$\begin{array}{c}\text { Altitude } \\
\text { (ft above msl) }\end{array}$} & \multirow{2}{*}{$\begin{array}{c}\text { Average } \\
\text { slope } \\
\text { (ft per } \\
\text { mile) } \\
\end{array}$} \\
\hline & Ranking order & & \begin{tabular}{|l|} 
above \\
mouth \\
\end{tabular} & \begin{tabular}{|l|l|} 
below \\
source
\end{tabular} & $\begin{array}{c}\text { at } \\
\text { site }\end{array}$ & $\begin{array}{c}\text { at } \\
\text { source }\end{array}$ & \\
\hline 219. & $\begin{array}{l}\text { Middle River Rouge above Red Run, } \\
\text { SE1/4 sec. } 6, \text { T.2 S., R. } 10 \text { E. } \\
\text { Red Run at mouth, }\end{array}$ & 100 & 4.2 & 27.0 & 596 & 933 & 12.5 \\
\hline 221 & $\begin{array}{l}\text { SE1/4 sec. } 6 \text {, T. } 2 \text { S., R. } 10 \text { E. } \\
\text { Middle River Rouge at Red Run, }\end{array}$ & 2.59 & 0 & 3.6 & 596 & 637 & 11.4 \\
\hline 222. & $\begin{array}{l}\text { SE1/4 sec. } 6, \text { T. } 2 \text { S., R. } 10 \text { E. } \\
\text { Middle River Rouge at Outer Drive, }\end{array}$ & 103 & 4.2 & 27.0 & 596 & 933 & 12.5 \\
\hline 223. & $\begin{array}{l}\mathrm{NE}^{1 / 4} \text { sec. 9, T. } 2 \text { S., R. } 10 \mathrm{E} . \\
\text { Middle River Rouge above Bills Drain, }\end{array}$ & 106 & 1.4 & 29.8 & 584 & 933 & 11.7 \\
\hline 224. & $\begin{array}{l}\mathrm{SE}^{1 / 4} / 4 \text { sec. } 9, \text { T. } 2 \text { S., R. } 10 \text { E. } \\
\text { Bills Drain at mouth, }\end{array}$ & 106 & 1.1 & 30.1 & 584 & 933 & 11.6 \\
\hline 225 . & $\begin{array}{l}\text { SE1/4 sec. 9, T.2 S., R. } 10 \mathrm{E} . \\
\text { Middle River Rouge at Bills Drain, }\end{array}$ & 2.96 & 0 & 5.2 & 584 & 638 & 10.4 \\
\hline 226. & $\begin{array}{l}\text { SE1/4 sec. 9, T.2 S., R. } 10 \mathrm{E} . \\
\text { Middle River Rouge at mouth, }\end{array}$ & 110 & 1.1 & 30.1 & 584 & 933 & 11.6 \\
\hline 227. & $\begin{array}{l}\text { Land Grant 615, T.2 S., R. } 10 \text { E. } \\
\text { River Rouge at Middle River Rouge, }\end{array}$ & 110 & 0 & 31.2 & 583 & 933 & 11.2 \\
\hline 228. & $\begin{array}{l}\text { Land Grant 615, T. } 2 \text { S., R. } 10 \text { E. } \\
\text { River Rouge at Ford Road, }\end{array}$ & 307 & 12.4 & 33.2 & 583 & 798 & 6.6 \\
\hline 229. & $\begin{array}{c}\text { Land Grant 615, T. } 2 \text { S., R. } 10 \text { E. } \\
\text { River Rouge above Lower River Rouge, } \\
\text { Land Grant 665, T. } 2 \text { S., R. } 10 \text { E. } \\
\text { Lower River Rouge above Parks Drain, }\end{array}$ & $\begin{array}{l}307 \\
308\end{array}$ & $\begin{array}{l}12.1 \\
10.2\end{array}$ & $\begin{array}{l}33.5 \\
35.4\end{array}$ & $\begin{array}{l}583 \\
575\end{array}$ & $\begin{array}{l}798 \\
798\end{array}$ & $\begin{array}{l}6.7 \\
6.3\end{array}$ \\
\hline 231. & $\begin{array}{l}\text { NW1/4 sec. } 19, \text { T.2 S., R. } 8 \text { E. } \\
\text { Parks Drain at mouth, }\end{array}$ & 3.99 & 22.3 & 3.8 & 717 & 860 & 37.6 \\
\hline 232. & $\begin{array}{l}\text { NW1/4 sec. 19, T.2 S., R. } 8 \text { E. } \\
\text { Lower River Rouge at Parks Drain, } \\
\text { NW1/4 sec 19 T 2 S. R } 8 \text { E }\end{array}$ & 1.59 & 223 & 3.6 & 717 & 809 & 25.6 \\
\hline 233. & $\begin{array}{c}\text { NW'1/4 sec. 19, T. } 2 \text { S., R. } 8 \text { E. } \\
\text { Lower River Rouge at Ridge Road, } \\
\text { SW11/4 sec. 19, T.2 S., R. } 8 \text { E. }\end{array}$ & 7.34 & 21.7 & 4.4 & 707 & 860 & $\begin{array}{l}37.6 \\
34.8\end{array}$ \\
\hline 235. & $\begin{array}{c}\text { SE }^{1 / 4} \text { sec. } 29, \text { T. } 2 \text { S., R. } 8 \text { E. } \\
\text { Lower River Rouge above Fowler Creek, }\end{array}$ & 9.01 & 19.2 & 6.9 & 680 & 860 & 26.1 \\
\hline 236. & $\begin{array}{c}\mathrm{SW}^{1 / 4} \text { sec. } 28, \mathrm{~T} .2 \mathrm{~S} ., \mathrm{R} .8 \mathrm{E} . \\
\text { Fowler Creek above Bazley and Foster Drain, } \\
\text { NW'1/4 sec. } 36 \text {, T.2 S., R. } 7 \text { E. }\end{array}$ & 4.84 & 19.1 & 5.6 & $\begin{array}{l}679 \\
719\end{array}$ & 860 & 25.8 \\
\hline 237. & $\begin{array}{l}\text { Bazley and Foster Drain at mouth, } \\
\text { NW1/4 sec. 36, T. } 2 \text { S., R. } 7 \text { E. }\end{array}$ & 2.60 & 0 & 2.6 & 719 & 760 & 15.8 \\
\hline 238. & $\begin{array}{c}\text { Fowler Creek at Bazley and Foster Drain, } \\
\text { NW11/4 sec. 36, T.2 S., R. } 7 \text { E. } \\
\text { Fowler Creek at Washtenaw County line, }\end{array}$ & 7.44 & 3.2 & 5.6 & 719 & 852 & 23.8 \\
\hline 240 . & $\begin{array}{l}\text { NE1/4 sec. 36, T. } 2 \text { S., R. } 7 \text { E. } \\
\text { Fowler Creek at Beck Road, }\end{array}$ & 7.62 & 2.5 & 6.3 & 707 & 852 & 23.0 \\
\hline 241. & $\begin{array}{l}\mathrm{SE}^{1 / 4} \text { sec. } 29, \mathrm{~T} .2 \mathrm{~S} ., \text { R. } 8 \mathrm{E} . \\
\text { Fowler Creek at mouth, }\end{array}$ & 12.0 & .1 & 8.7 & 680 & 852 & 19.8 \\
\hline 242. & $\begin{array}{l}\text { SW11/4 sec. } 28 \text {, T. } 2 \text { S., R. } 8 \text { E. } \\
\text { Lower River Rouge at Fowler Creek, }\end{array}$ & 12.0 & 0 & 8.8 & $679=$ & 852 & 19.6 \\
\hline 240. & NW1/4 sec. 27 , T. 2 S., R. 8 E. & 22.4 & 16.9 & 9.2 & 665 & 860 & 21.2 \\
\hline 244. & $\begin{array}{l}\text { Mott Drain at mouth, } \\
\text { NW11/4 sec. } 27, \text { T. } 2 \text { S., R. } 8 \text { E. }\end{array}$ & 2.08 & 0 & 3.1 & 665 & 704 & 12.6 \\
\hline 245. & $\begin{array}{l}\text { Lower River Rouge at Mott Drain, } \\
\text { NW11/4 sec. 27, T.2 S., R. } 8 \text { E. }\end{array}$ & 24.5 & 16.9 & 9.2 & 665 & 860 & 21.2 \\
\hline 246. & $\begin{array}{l}\text { Lower River Rouge above Sines Drain, } \\
\text { SE1/4 sec. } 27 \text {, T.2 S., R. } 8 \text { E. } \\
\text { Sines Drain above Apple Run. }\end{array}$ & 24.7 & 16.5 & 9.6 & 662 & 860 & 20.6 \\
\hline 248. & $\begin{array}{c}\text { SW1/4 sec. 33, T. } 2 \text { S., R. } 8 \text { E. } \\
\text { Apple Run above Horner Drain, }\end{array}$ & 1.44 & 2.3 & 3.1 & 683 & 715 & 10.3 \\
\hline 249. & $\begin{array}{l}\text { NW1/4 sec. } 4 \text {, T. } 3 \text { S., R. } 8 \text { E. } \\
\text { Horner Drain at mouth. }\end{array}$ & .67 & .9 & 1.7 & 687 & 695 & 4.7 \\
\hline & NW1/4 sec. 4, T. 3 S., R. 8 E. & 4.36 & 0 & 1.4 & 687 & 700 & 9.3 \\
\hline
\end{tabular}


PHYSIOGRAPHIC DATA FOR SELECTED LOCATIONS IN THE RIVER ROUGE BASIN

\begin{tabular}{|c|c|c|c|c|c|c|c|}
\hline \multirow{2}{*}{$\begin{array}{l}\text { Num- } \\
\text { ber } \\
\text { Key }\end{array}$} & Stream and location & \multirow{2}{*}{$\begin{array}{l}\text { Drainage } \\
\text { area } \\
\text { (sq mi) }\end{array}$} & \multicolumn{2}{|c|}{ Miles } & \multicolumn{2}{|c|}{$\begin{array}{c}\text { Altitude } \\
\text { (ft above msl) }\end{array}$} & \multirow{2}{*}{$\begin{array}{l}\text { Average } \\
\text { slope } \\
\text { (ft per } \\
\text { mile) }\end{array}$} \\
\hline & Ranking order & & $\begin{array}{l}\text { above } \\
\text { mouth }\end{array}$ & \begin{tabular}{|l} 
below \\
source
\end{tabular} & $\begin{array}{c}\text { at } \\
\text { site }\end{array}$ & $\begin{array}{c}\text { at } \\
\text { source }\end{array}$ & \\
\hline 250. & $\begin{array}{l}\text { Apple Run at Horner Drain, } \\
\text { NW11/4 sec. 4, T. } 3 \text { S., R. } 8 \text { E. }\end{array}$ & 5.03 & .9 & 1.7 & 687 & 695 & 4.7 \\
\hline 251. & $\begin{array}{l}\text { Apple Run at mouth, } \\
\text { SW1/4 sec. } 33 \text {, T. } 2 \text { S., R. } 8 \text { E. } \\
\text { Sines Drain at Apple Run, }\end{array}$ & 6.21 & 0 & 2.6 & 683 & 695 & 4.6 \\
\hline 253. & $\begin{array}{l}\text { SW1/4 sec. 33, T. } 2 \text { S., R. } 8 \text { E. } \\
\text { Sines Drain at mouth, }\end{array}$ & 7.65 & 2.3 & 3.1 & 683 & 715 & 10.3 \\
\hline 254. & $\begin{array}{l}\text { SE1/4 sec. } 27, \text { T. } 2 \text { S., R. } 8 \text { E. }^{\text {Lower River Rouge at Sines Drain, }}\end{array}$ & 9.13 & 0 & 5.4 & 662 & 715 & 9.8 \\
\hline 255. & $\begin{array}{l}\mathrm{SE} 1 / 4 \text { sec. } 27, \text { T. } 2 \mathrm{~S} ., \mathrm{R} .8 \mathrm{E} \\
\text { Lower River Rouge above McKinstry Drain, }\end{array}$ & 33.8 & 16.5 & 9.6 & 662 & 860 & 20.6 \\
\hline 256. & $\begin{array}{l}\mathrm{SW}^{1 / 4} \mathrm{sec} .26, \mathrm{~T} .2 \mathrm{~S} ., \mathrm{R} .8 \mathrm{E} . \\
\text { McKinstry Drain at mouth, }\end{array}$ & 34.2 & 15.5 & 10.6 & 656 & 860 & 19.2 \\
\hline 257. & $\begin{array}{l}\text { SW } 1 / 4 \mathrm{sec} .26, \mathrm{~T} .2 \mathrm{~S} ., \text { R. } 8 \mathrm{E} \\
\text { Lower River Rouge at McKinstry Drain, }\end{array}$ & 2.82 & 0 & 3.4 & 656 & 694 & 11.2 \\
\hline 258. & $\begin{array}{l}\text { SW1/4 sec. 26, T.2 S., R. } 8 \text { E. } \\
\text { Lower River Rouge at Lotz Road, }\end{array}$ & 37.0 & 15.5 & 10.6 & 656 & 860 & 19.2 \\
\hline 259. & $\begin{array}{c}\text { SW1/4 sec. 25, T. 2 S., R. } 8 \text { E. } \\
\text { Lower River Rouge above Fellows Creek, } \\
\text { SE }^{1 / 4} \text { sec. 25, T. 2 S. R. } 8 \text { E. }\end{array}$ & 38.6 & 13.9 & 12.2 & 645 & 860 & 17.6 \\
\hline 260. & $\begin{array}{c}\text { Fellows Creek above Ingall Drain, } \\
\text { SW1/4 sec. 31, T. } 1 \text { S., R. } 8 \text { E. } \\
\text { Ingall Drain at mouth. }\end{array}$ & 1.30 & 10.1 & 1.3 & 803 & 868 & 50.0 \\
\hline 262. & $\begin{array}{l}\text { SW11/4 sec. 31, T. } 1 \text { S., R. } 8 \text { E. }^{\text {Fellows Creek at Ingall Drain, }}\end{array}$ & 1.26 & 0 & 2.4 & 803 & 847 & 18.3 \\
\hline 263. & $\begin{array}{l}\text { SW1/4 sec. 31, T. } 1 \text { S., R. } 8 \text { E. } \\
\text { Fellows Creek at Beck Road, }\end{array}$ & 2.56 & 10.1 & 1.3 & 803 & 868 & 50.0 \\
\hline 264. & $\begin{array}{l}\text { SE } 1 / 4 \text { sec. } 8 \text {, T. } 2 \text { S., R. } 8 \text { E. } \\
\text { Fellows Creek above North Branch Fellows } \\
\text { Creek, }\end{array}$ & 6.57 & 5.8 & 5.6 & 697 & 868 & 30.5 \\
\hline 265. & $\begin{array}{l}\text { SW1/4 sec. 10, T. } 2 \text { S., R. } 8 \text { E. } \\
\text { North Branch Fellows Creek at mouth, }\end{array}$ & 6.89 & 4.8 & 6.6 & 684 & 868 & 27.9 \\
\hline 266. & $\begin{array}{l}\text { SW1/4 sec. 10, T.2 S., R. } 8 \text { E. } \\
\text { Fellows Creek at North Branch Fellows } \\
\text { Creek, }\end{array}$ & 2.46 & 0 & 4.0 & 684 & 834 & 37.5 \\
\hline 268. & $\begin{array}{l}\text { SW1/4 sec. 10, T. } 2 \text { S., R. } 8 \text { E. } \\
\text { Fellows Creek above Green Drain, } \\
\text { SW1/4 sec. 14, T.2 S., R. } 8 \text { E. } \\
\text { Green Drain at mouth, }\end{array}$ & 9.35 & $\begin{array}{l}4.8 \\
3.4\end{array}$ & 6.6 & $\begin{array}{l}684 \\
670\end{array}$ & $\begin{array}{l}868 \\
868\end{array}$ & 27.9 \\
\hline 269. & $\begin{array}{l}\mathrm{SW}^{1 / 4} \text { sec. 14, T.2 S., R. } 8 \mathrm{E} . \\
\text { Fellows Creek at Green Drain, }\end{array}$ & 1.65 & 0 & 3.0 & 670 & 709 & 13.0 \\
\hline 270 . & $\begin{array}{l}\text { SW1/4 sec. 14, T. } 2 \text { S., R. } 8 \text { E. } \\
\text { Fellows Creek above Hustin Drain, }\end{array}$ & 11.8 & 3.4 & 8.0 & 670 & 868 & 24.8 \\
\hline 271. & $\begin{array}{l}\mathrm{NW}^{1 / 4} / 4 \text { sec. } 24, \mathrm{~T} .2 \mathrm{~S} ., \mathrm{R} .8 \mathrm{E} . \\
\text { Hustin Drain at mouth, }\end{array}$ & 12.4 & 1.9 & 9.5 & 659 & 868 & 22.0 \\
\hline 272. & $\begin{array}{l}\text { NW1/4 sec. } 24, \text { T. } 2 \text { S., R. } 8 \text { E. } \\
\text { Fellows Creek at Hustin Drain. }\end{array}$ & 1.21 & 0 & 2.7 & 659 & 684 & 9.3 \\
\hline 212. & NW1/4 sec. 24, T. 2 S., R. 8 E. & 13.6 & 1.9 & 9.5 & 659 & 868 & 22.0 \\
\hline 273. & $\begin{array}{l}\text { Fellows Creek above Truesdell Drain, } \\
\text { NW11/4 sec. 24, T. } 2 \text { S., R. } 8 \text { E. } \\
\text { Truesdell Drain at mouth. }\end{array}$ & 13.7 & 1.5 & 9.9 & 655 & 868 & 21.5 \\
\hline 275. & $\begin{array}{l}\text { NW1/4 sec. } 24 \text {, T. } 2 \text { S., R. } 8 \text { E. } \\
\text { Fellows }\end{array}$ & .70 & 0 & 2.0 & 655 & 674 & 9.5 \\
\hline 276. & $\begin{array}{l}\text { NW1/4 sec. } 24 \text {, T. } 2 \text { S., R. } 8 \text { E. } \\
\text { Fellows Creek at Palmer Road, }\end{array}$ & 14.4 & 1.5 & 9.9 & 655 & 868 & 21.5 \\
\hline & SE $1 / 4$ sec. 24, T. 2 S., R. 8 E. & 16.0 & .9 & 10.5 & 650 & 868 & 20.8 \\
\hline 277. & $\begin{array}{l}\text { Fellows Creek at mouth, } \\
\text { SE1/4 sec. } 25, \text { T.2 S., R. } 8 \text { E. }\end{array}$ & 16.7 & 0 & 11.4 & 644 & 868 & 19.6 \\
\hline 278. & $\begin{array}{l}\text { Lower River Rouge at Fellows Creek, } \\
\mathrm{SE}^{1 / 4} \text { sec. } 25 \text {, T. } 2 \text { S., R. } 8 \text { E. }\end{array}$ & 55.5 & 13.6 & 12.5 & 644 & 860 & 17.3 \\
\hline 279. & $\begin{array}{l}\text { Lower River Rouge above Hannan Drain, } \\
\mathrm{SW}^{1 / 4} \text { sec. } 30, \text { T. } 2 \text { S., R. } 9 \text { E. }\end{array}$ & 55.6 & 13.3 & 12.8 & 642 & 860 & 17.0 \\
\hline
\end{tabular}


PHYSIOGRAPHIC DATA FOR SELECTED LOCATIONS IN THE RIVER ROUGE BASIN

\begin{tabular}{|c|c|c|c|c|c|c|c|}
\hline \multirow{2}{*}{$\begin{array}{l}\text { Num- } \\
\text { ber } \\
\text { Key }\end{array}$} & Stream and location & \multirow{2}{*}{$\begin{array}{c}\text { Drainage } \\
\text { area } \\
(\mathrm{sq} \mathrm{mi})\end{array}$} & \multicolumn{2}{|c|}{ Miles } & \multicolumn{2}{|c|}{$\begin{array}{c}\text { Altitude } \\
\text { (ft above msl) }\end{array}$} & \multirow{2}{*}{$\begin{array}{l}\text { Average } \\
\text { slope } \\
\text { (ft per } \\
\text { mile) }\end{array}$} \\
\hline & Ranking order & & $\begin{array}{l}\text { above } \\
\text { mouth }\end{array}$ & $\begin{array}{l}\text { below } \\
\text { source }\end{array}$ & $\begin{array}{c}\text { at } \\
\text { site }\end{array}$ & $\begin{array}{c}\text { at } \\
\text { source }\end{array}$ & \\
\hline 280. & $\begin{array}{l}\text { Hannan Drain at mouth, } \\
\text { SW1/4 sec. 30, T.2 S., R. } 9 \text { E. }\end{array}$ & .59 & 0 & 1.6 & 642 & 664 & 13.8 \\
\hline 281. & $\begin{array}{l}\text { Lower River Rouge at Hannan Drain, } \\
\text { SW11/4 sec. } 30 \text {, T. } 2 \text { S., R. } 9 \text { E. }\end{array}$ & 56.2 & 13.3 & 12.8 & 642 & 865 & 17.0 \\
\hline 282. & 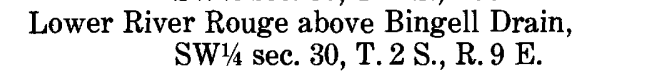 & 56.3 & 13.0 & 13.1 & 641 & 860 & 16.7 \\
\hline 283. & $\begin{array}{l}\text { Bingell Drain above Bell Drain, } \\
\mathrm{SE}^{1 / 4} \text { sec. } 36 \text {, T. } 2 \text { S., R. } 8 \text { E. }\end{array}$ & .50 & .9 & 1.3 & 660 & 669 & 6.9 \\
\hline 284. & $\begin{array}{l}\text { Bell Drain at mouth, } \\
\mathrm{SE}^{1 / 4} \text { sec. } 36, \text { T. } 2 \text { S., R. } 8 \text { E. }\end{array}$ & 1.04 & 0 & 2.1 & 660 & 674 & 6.7 \\
\hline 285. & $\begin{array}{l}\text { Bingell Drain at Bell Drain, } \\
\text { SE } 1 / 4 \text { sec. } 36 \text {, T. } 2 \text { S., R. } 8 \text { E. }\end{array}$ & 1.54 & .9 & 1.3 & 660 & 669 & 6.9 \\
\hline 286. & $\begin{array}{l}\text { Wilbur Drain at mouth, } \\
\mathrm{SE}^{1 / 4} \text { sec. } 36, \mathrm{~T} .2 \mathrm{~S} ., \text { R. } 8 \text { E. }\end{array}$ & .30 & 0 & 1.3 & 660 & 669 & 6.9 \\
\hline 287. & 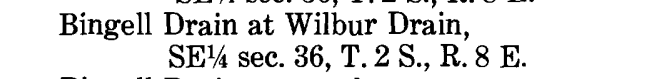 & 1.84 & .9 & 1.3 & 660 & 669 & 6.9 \\
\hline 288. & $\begin{array}{l}\text { Bingell Drain at mouth, } \\
\text { SW } 1 / 4^{4} \text { sec. } 30, \text { T. } 2 \text { S., R. } 9 \text { E. }\end{array}$ & 2.30 & 0 & 2.2 & 641 & 669 & 12.7 \\
\hline 289. & 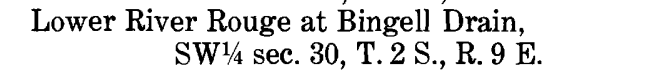 & 58.6 & 13.0 & 13.1 & 641 & 860 & 16.7 \\
\hline 290. & $\begin{array}{l}\text { Lower River Rouge above Hunter Drain, } \\
\text { SW1/4 sec. } 29 \text {, T. } 2 \text { S., R. } 9 \text { E. }\end{array}$ & 59.3 & 11.8 & 14.3 & 634 & 860 & 15.8 \\
\hline 291. & $\begin{array}{l}\text { Hunter Drain at mouth, } \\
\text { SW11/4 sec. } 29, \text { T. } 2 \text { S., R. } 9 \text { E. }\end{array}$ & 3.50 & 0 & 2.8 & 634 & 668 & 12.1 \\
\hline 292. & $\begin{array}{l}\text { Lower River Rouge at Hunter Drain, } \\
\text { SW }^{1 / 4} \text { sec. } 29 \text {, T. } 2 \text { S., R. } 9 \text { E. }\end{array}$ & 62.8 & 11.8 & 14.3 & 634 & 860 & 15.8 \\
\hline 293. & 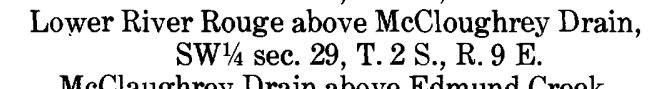 & 62.8 & 11.6 & 14.5 & 633 & 860 & 15.7 \\
\hline 294. & $\begin{array}{l}\text { McClaughrey Drain above Edmund Creek, } \\
\text { NW11/4 sec. 32, T. } 2 \text { S., R.9 E. } \\
\text { Edmund Creek at mouth, }\end{array}$ & 6.39 & .4 & 6.6 & 646 & 694 & 7.3 \\
\hline $\begin{array}{l}296 . \\
297 .\end{array}$ & $\begin{array}{l}\text { NW1/4 sec. 32, T.2 S., R. } 9 \mathrm{E} . \\
\text { McClaughrey Drain at Edmund Creek, } \\
\text { NW11/4 sec. } 32 \text {, T. } 2 \text { S., R. } 9 \mathrm{E} \text {. } \\
\text { McClaughrey Drain at mouth, }\end{array}$ & 7.03 & 0 & 6.6 & $\begin{array}{l}646 \\
646\end{array}$ & 694 & $\begin{array}{r}12.1 \\
7.3\end{array}$ \\
\hline 290. & 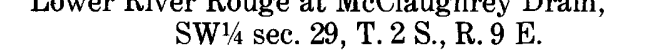 & 70.0 & 11.6 & 14.5 & 633 & 860 & 15.7 \\
\hline 299. & $\begin{array}{l}\text { Lower River Rouge at Wayne Road, } \\
\text { SW11/4 sec. } 28 \text {, T.2 S., R. } 9 \text { E. }\end{array}$ & 71.2 & 10.3 & 15.8 & 630 & 860 & 14.6 \\
\hline 300. & $\begin{array}{l}\text { Lower River Rouge above Boice Drain, } \\
\text { NW11/4 sec. } 26 \text {, T. } 2 \text { S., R. } 9 \text { E. }\end{array}$ & 73.1 & 7.5 & 18.6 & 615 & 860 & 13.2 \\
\hline 301. & $\begin{array}{l}\text { Boice Drain at mouth, } \\
\text { NW1/4 sec. } 26, \text { T. } 2 \text { S., R. } 9 \text { E. }\end{array}$ & 3.58 & 0 & 4.0 & 615 & 664 & 12.2 \\
\hline 302. & $\begin{array}{l}\text { Lower River Rouge at Boice Drain, } \\
\text { NW1/4 sec. } 26 \text {, T. } 2 \text { S., R.9 E. }\end{array}$ & 76.7 & 7.5 & 18.6 & 615 & 860 & 13.2 \\
\hline 303. & $\begin{array}{l}\text { Lower River Rouge above Carver Drain, } \\
\text { SW11/4 sec. 19, T. } 2 \text { S., R. } 10 \text { E. }\end{array}$ & 79.1 & 5.1 & 21.0 & 601 & 860 & 12.3 \\
\hline 304. & $\begin{array}{l}\text { Carver Drain at mouth, } \\
\text { SW11/4 sec. 19, T. } 2 \text { S., R. } 10 \text { E. }\end{array}$ & 1.15 & 0 & 3.1 & 601 & 644 & 13.9 \\
\hline 305. & $\begin{array}{l}\text { Lower River Rouge at Carver Drain, } \\
\text { SW1/4 sec. 19, T. } 2 \text { S., R. } 10 \mathrm{E} .\end{array}$ & 80.2 & 5.1 & 21.0 & 601 & 860 & 12.3 \\
\hline 306. & $\begin{array}{l}\text { Lower River Rouge above Perrin Drain, } \\
\text { SW } 1 / 4 \text { sec. } 19, \text { T.2 S., R. } 10 \text { E. }\end{array}$ & 80.3 & 4.7 & 21.4 & 598 & 860 & 12.2 \\
\hline 307. & $\begin{array}{l}\text { Perrin Drain at mouth, } \\
\text { SW } 1 / 4 \text { sec. } 19 \text {, T. } 2 \text { S., R. } 10 \text { E. }\end{array}$ & 2.79 & 0 & 5.1 & 598 & 664 & 12.9 \\
\hline 308. & $\begin{array}{l}\text { Lower River Rouge at Perrin Drain, } \\
\text { SW11/4 sec. 19, T. } 2 \text { S., R. } 10 \text { E. }\end{array}$ & 83.1 & 4.7 & 21.4 & 598 & 860 & 12.2 \\
\hline 309. & $\begin{array}{l}\text { Lower River Rouge at John Daily Road (gage), } \\
\mathrm{SE}^{1 / 4} \text { sec. } 19 \text {, T. } 2 \text { S., R. } 10 \text { E. }\end{array}$ & 83.2 & 4.4 & 21.7 & 597 & 860 & 12.1 \\
\hline 310. & $\begin{array}{l}\text { Lower River Rouge above Bahn Drain, } \\
\mathrm{SE}^{1 / 4} \text { sec. } 20 \text {, T. } 2 \text { S., R.10 E. }\end{array}$ & 84.6 & 2.9 & 23.2 & 588 & 860 & 11.7 \\
\hline
\end{tabular}


PHYSIOGRAPHIC DAIA FOR SELECTED LOCATIONS IN THE RIVER ROUGE BASIN

\begin{tabular}{|c|c|c|c|c|c|c|c|}
\hline \multirow{2}{*}{$\begin{array}{c}\text { Num } \\
\text { ber } \\
\text { Key }\end{array}$} & \multirow[t]{2}{*}{ Stream and location } & \multirow{2}{*}{$\begin{array}{l}\text { Drainage } \\
\text { area } \\
\text { (sq mi) }\end{array}$} & \multicolumn{2}{|c|}{ Miles } & \multicolumn{2}{|c|}{$\begin{array}{c}\text { Altitude } \\
\text { (ft above msl) }\end{array}$} & \multirow{2}{*}{\begin{tabular}{|c} 
Average \\
slope \\
(ft per \\
mile)
\end{tabular}} \\
\hline & & & $\begin{array}{l}\text { above } \\
\text { mouth }\end{array}$ & $\begin{array}{l}\text { below } \\
\text { source }\end{array}$ & $\begin{array}{l}\text { at } \\
\text { site }\end{array}$ & $\begin{array}{c}\text { at } \\
\text { source }\end{array}$ & \\
\hline $\begin{array}{l}311 . \\
312 . \\
313 . \\
314 . \\
315 . \\
316 . \\
317 . \\
318 . \\
319 .\end{array}$ & $\begin{array}{c}\text { Bahan Drain at mouth, } \\
\text { SE1/4 sec. } 20, \text { T. } 2 \text { S., R. } 10 \text { E. } \\
\text { Lower River Rouge at Bahan Drain, } \\
\text { SE } 1 / 4 \text { sec. } 20, \text { T. } 2 \text { S., R. } 10 \text { E. } \\
\text { Lower River Rouge at Outer Drive, } \\
\text { SW } 1 / 4 \text { sec. } 21 \text {, T. } 2 \text { S., R. } 10 \text { E. } \\
\text { Lower River Rouge at Ford Field, } \\
\text { Land Grant } 665 \text {, T. } 2 \text { S., R. } 10 \text { E. } \\
\text { Lower River Rouge at South Brady Street, } \\
\text { Land Grant } 665, \text { T. } 2 \text { S., R. } 10 \text { E. } \\
\text { Lower River Rouge at mouth, } \\
\text { Land Grant } 665, \text { T. } 2 \text { S., R. } 10 \text { E. } \\
\text { River Rouge at Lower River Rouge, } \\
\text { Land Grant } 665, \text { T. } 2 \text { S., R. } 10 \text { E. } \\
\text { River Rouge Canal, } \\
\text { Land Grant 496, T. } 2 \text { S., R. } 11 \text { E. } \\
\text { River Rouge at mouth, } \\
\text { Land Grant 45, T. } 2 \text { S., R. } 11 \text { E. }\end{array}$ & $\begin{array}{r}2.67 \\
87.3 \\
90.4 \\
91.9 \\
91.9 \\
92.3\end{array}$ & $\begin{array}{l}0 \\
2.9 \\
2.2 \\
1.0 \\
.8 \\
0\end{array}$ & $\begin{array}{r}3.0 \\
23.2 \\
23.9 \\
25.1 \\
25.3 \\
26.1 \\
\end{array}$ & $\begin{array}{l}588 \\
588 \\
585 \\
577 \\
576 \\
575 \\
\\
\\
574 \\
574\end{array}$ & $\begin{array}{l}630 \\
860 \\
860 \\
860 \\
860 \\
860\end{array}$ & $\begin{array}{r}14.0 \\
11.7 \\
11.5 \\
11.3 \\
11.2 \\
10.9 \\
\\
5.1 \\
4.9\end{array}$ \\
\hline
\end{tabular}

